\title{
Monitoramento da biodeterioração da madeira de três espécies amazônicas pela técnica da colorimetria
}

Diego Martins STANGERLIN ${ }^{1}$, Alexandre Florian da COSTA², Joaquim Carlos GONÇALEZ ${ }^{2}$, Tereza Cristina Monteiro PASTORE ${ }^{3}$, Alencar GARLET ${ }^{3}$

\begin{abstract}
RESUMO
Objetivou-se avaliar o emprego da colorimetria na caracterização da biodeterioração das madeiras de marupá, jequitibá e cumaru submetidas ao ataque de fungos de podridáo branca e parda. Para tanto, corpos de prova de cada espécie amazônica foram submetidos a ensaios de apodrecimento acelerado, de acordo com a American Society for Testing and Materials - ASTM D2017, durante 20 semanas. Os parâmetros colorimétricos L* (luminosidade), a* (coordenada verde-vermelho), b* (coordenada amarelo-azul) e a variação total da cor $(\Delta \mathrm{E})$ foram determinados semanalmente até a quarta semana, e posteriormente a cada duas semanas até a vigésima semana, com auxílio de um espectrofotocolorímetro. Adicionalmente, foram determinadas as perdas de massa dos corpos de prova. Os resultados permitiram destacar que a colorimetria foi eficaz no monitoramento da biodeterioração da madeira, bem como para diferenciação da podridão branca e parda. As melhores prediçóes da resistência natural aos fungos de podridão branca e parda foram obtidas com os parâmetros $b^{*}$ e $L^{*}$, respectivamente. Enfatiza-se também, o emprego da $\Delta \mathrm{E}$ como parâmetro auxiliar na estimativa da biodeterioração da madeira, em razáo de suas correlaçóes significativas com a perda de massa.
\end{abstract}

PALAVRAS-CHAVE: durabilidade natural, parâmetros colorimétricos, podridão branca, podridão parda, madeiras tropicais.

\section{Monitoring of biodeterioration of three Amazonian wood species by the colorimetry technique}

\section{ABSTRACT}

This work aimed to evaluate the use of colorimetry in the characterization of biodeterioration marupá, jequitibá and cumaru wood, submitted to white and brown rot. For this purpose, specimens of each Amazonian species were submitted to accelerated decay tests, according to American Society for Testing and Materials - ASTM D2017, during 20 weeks. With a colorimeter, the colorimetric parameters $\mathrm{L}^{*}$ (lightness), $\mathrm{a}^{*}$ (green-red coordinate), $\mathrm{b}^{*}$ (blue-yellow coordinate) and total color change $(\Delta \mathrm{E})$ were determined weekly until the fourth week, and then every two weeks until the twentieth week. Additionally, it was determined the mass loss. The results indicated that the colorimetry was effective in monitoring of biodeterioration of wood, as well as to differentiate white and brown rot. The best predictions of natural resistance to white and brown rot were obtained with the parameters $\mathrm{b}^{*}$ and $\mathrm{L}^{*}$, respectively. We also emphasizes the use of $\Delta \mathrm{E}$ to estimate the biodeterioration of wood, because of their significant correlations with weight loss.

KEYWORDS: natural durability, colorimetric parameters, white rot, brown rot, tropical wood.

\footnotetext{
Instituto de Ciências Agrárias e Ambientais, Universidade Federal de Mato Grosso, Avenida Alexandre Ferronato, 1200, Setor Industrial, 78550-000, Sinop, MT, Brasil. Email: stangerlin@ufmt.br

2 Departamento de Engenharia Florestal, Faculdade de Tecnologia, Universidade de Brasilia, CxP. 04357, 70904-970, Brasília, DF, Brasil. Email: lucate@unb.br; gonçalez@unb.br

${ }^{3}$ Laboratório de Produtos Florestais, Serviço Florestal Brasileiro, L4 Norte - SCEN, Trecho 2, Lote 4, Bloco B, 70818-900, Brasília, DF, Brasil. Email: tereza.pastore@gmail.com; alencar.garlet@florestal.gov.br
} 


\section{INTRODUÇÃO}

Dependendo do local e modo de emprego, a madeira pode ser deteriorada por uma gama de agentes biológicos, dentre os quais estão incluídos os fungos, os insetos, as bactérias e os organismos marinhos (Santini 1988; Zabel e Morrell 1992; Eaton e Hale 1993). Em razáo da frequência de sua ação, os principais agentes biológicos são os fungos, em especial os apodrecedores (Zabel e Morrell 1992; Carneiro et al. 2009).

Entre os fungos responsáveis pelo apodrecimento dos materiais lignocelulósicos destaca-se a classe dos Basidiomicetos na qual se encontram os fungos responsáveis pela podridão parda e branca, que possuem características enzimáticas próprias, quanto à deterioração dos constituintes químicos (Santini 1988). Os primeiros deterioram os polissacarídeos da parede celular, enquanto os últimos atacam, indistintamente, tanto os polissacarídeos quanto a lignina (Zabel e Morrell 1992).

A resistência natural a um determinado tipo de fungo é, frequentemente, caracterizada, apenas, por meio da perda de massa. De acordo com Zabel e Morrell (1992), Eaton e Hale (1993) e Stangerlin (2012) deve-se ressaltar que a perda de massa, por vezes, não apresenta a mesma proporção de alteração que as demais propriedades da madeira (físicas, químicas e mecânicas), sendo desse modo de pouca utilidade quando avaliada isoladamente.

Dentre os ensaios que tem por finalidade a caracterização de alteraçôes superficiais da madeira pode-se destacar a colorimetria, a qual é definida como sendo o ramo da ciência (física ótica e geométrica) que tem por objetivo a medição de cores (Gonçalez 1993). O mesmo autor ainda afirmou que a atribuição de uma determinada cor, apenas, pela análise visual de um objeto é subjetiva, visto que a mesma pode diferenciar de acordo com o observador e a fonte de luminosidade. Para contornar essa subjetividade foram desenvolvidos métodos quantitativos que empregam variáveis numéricas para as interaçôes provocadas pela luz numa superfície (Stangerlin 2012).

Dentre os métodos quantitativos de medição de cores pode-se destacar o CIELab, recomendado pela Comission International de L'Eclairage, o qual se caracteriza por definir a sensação da cor em razão de três elementos: a luminosidade, a matiz e a cromaticidade (Camargos e Gonçalez 2001).

O emprego da colorimetria, por meio do sistema CIELab, tem apresentado viabilidade na classificaçáo da qualidade da madeira, como por exemplo, o agrupamento de madeiras com propriedades mecânicas (Moya e Marín 2011) e físicas (Nishino 2000) similares.

Segundo Fengel e Wegener (1984), a cor da madeira deriva da composiçẫo química das substâncias presentes no xilema, em especial os compostos fenólicos. As características gerais (textura, grã e figura) e os planos de orientação (transversal, longitudinal-tangencial e longitudinal-radial) também influenciam diretamente na cor da madeira (Camargos e Gonçalez 2001).

Além da diversidade de suas características intrínsecas (químicas e anatômicas), a madeira pode apresentar alteração da cor em razão de efeitos externos. Sendo assim, diversos estudos tem apresentado a viabilidade da colorimetria na caracterização da madeira submetida a processos de intemperismo artificial (Martins et al. 2011), a diferentes condiçôes de secagem (Tovar et al. 2009), a tratamentos térmicos (Unsal et al. 2003) e a aplicação de produtos de acabamento (Valverde e Moya 2010).

Da mesma maneira, a ação de fungos apodrecedores causa alteraçôes nos parâmetros colorimétricos da madeira à medida que é deteriorada, no entanto os estudos tem focado, apenas, nos estágios finais de deterioraçấo (Morais e Costa 2007; Souza et al. 2010; Costa et al. 2011; Almeida et al. 2012).

A adoção de técnicas que permitam o reconhecimento do ataque de fungos apodrecedores em estágios iniciais ou intermediários, por vezes é imperiosa. Nesse sentido, objetivouse avaliar o emprego da colorimetria na caracterização da biodeterioração das madeiras de marupá, jequitibá e cumaru submetidas ao ataque de fungos de podridão branca e parda.

\section{MATERIAL E MÉTODOS}

Para realização desse estudo utilizaram-se seis pranchas, com dimensôes nominais de $0,04 \times 0,25 \times 3 \mathrm{~m}$ (espessura $\mathrm{x}$ largura $\mathrm{x}$ comprimento), de três espécies amazônicas, marupá (Simarouba amara Aubl., Simaroubaceae), jequitibá (Cariniana micrantha Ducke, Lecythidaceae) e cumaru (Dipteryx odorata (Aubl.) Willd., Fabaceae), sendo as mesmas obtidas, de forma aleatória, em estabelecimentos madeireiros do Distrito Federal (DF).

As pranchas de cada espécie amazônica foram anatomicamente identificadas na Área de Anatomia e Morfologia da Madeira do Laboratório de Produtos Florestais (LPF) - Serviço Florestal Brasileiro (SFB), em Brasília, DF. Posteriormente, procedeu-se à confecção dos corpos de prova, da região do cerne, com dimensões nominais de 2,5 x 2,5 x $0,9 \mathrm{~cm}$, sendo a menor dimensão no sentido axial, para os ensaios de apodrecimento acelerado.

Os corpos de prova foram submetidos a ensaios de apodrecimento acelerado em laboratório com dois tipos de fungos apodrecedores, sendo um de podridão parda (Gloeophyllum trabeum (Pers.; Fr.) Murrill) e outro de podridão branca (Trametes versicolor (L.; Fr.) Pilát). Desse modo, os tratamentos foram estabelecidos na combinação das três espécies amazônicas e dois tipos de fungos, totalizando seis tratamentos, sendo utilizados 144 corpos de prova 
por tratamento. Adicionalmente, para cada espécie, foram avaliados blocos de correção (avaliaçáo da perda de massa operacional), sem ataque de fungos, com 144 corpos de prova.

O ensaio de apodrecimento acelerado realizou-se na Área de Biodegradação e Preservação da Madeira do LPF/SFB ao empregar metodologia adaptada do procedimento D2017 da ASTM (2005).

Em frascos de vidro com tampa rosqueável e capacidade de $150 \mathrm{~mL}$ foram adicionados $70 \mathrm{~g}$ de solo de horizonte B (peneirado em malha de $30 \mathrm{~mm}$ de abertura e com $\mathrm{pH}$ corrigido para 6,0) e $20 \mathrm{~mL}$ de água destilada. Posteriormente, foi inserida uma placa suporte do alburno de madeiras de Cecropia sp. ou de Pinus sp., para desenvolvimento dos fungos de podridáo branca e parda, respectivamente, as quais foram dispostas sobre o solo. Por fim, os frascos foram autoclavados em temperatura de $120^{\circ} \mathrm{C}$ e pressão de 1 atm durante 1 hora.

$\mathrm{O}$ meio de cultura foi preparado à base de extrato de malte líquido, e posteriormente, procedeu-se a autoclavagem do mesmo, nas condiçôes citadas, durante 20 minutos. Os inóculos dos fungos, de aproximadamente $1 \mathrm{~cm}^{2}$, foram adicionados ao meio de cultura líquido, em seguida o material permaneceu em câmara de incubação, com temperatura de 27 ${ }^{\circ} \mathrm{C}$ e umidade relativa de $70 \%$, por um período de 15 dias, para o completo desenvolvimento dos fungos.

Após o período de incubação prévia, o meio de cultura foi transferido para o copo de um liquidificador esterilizado, em condiçóes assépticas, sendo levemente batido, de modo a proporcionar a distribuição homogênea dos micélios dos fungos. A inoculação foi realizada de modo que em cada frasco fossem adicionados, sobre a placa suporte e o solo, aproximadamente, $3 \mathrm{~mL}$ do meio de cultura. Em sequencia, os frascos permaneceram em câmara de incubação sob condiçôes citadas, até o completo recobrimento do micélio sobre a placa suporte, aproximadamente, 30 dias.

Por fim, adicionou-se um corpo de prova de madeira em cada frasco, que, antes disso, tiveram sua massa obtida ao permanecerem em estufa de circulação forçada de ar a $50{ }^{\circ} \mathrm{C}$, até atingirem massa constante, e esterilizados em autoclave a $120^{\circ} \mathrm{C}$ durante 40 minutos. Os corpos de prova permaneceram em contato com os fungos durante 20 semanas na câmara de incubação.

Durante os ensaios de apodrecimento foram realizadas amostragens dos corpos de prova deteriorados nos seguintes períodos de tempo, $1^{\mathrm{a}}, 2^{\mathrm{a}}, 3^{\mathrm{a}}, 4^{\mathrm{a}}, 6^{\mathrm{a}}, 8^{\mathrm{a}}, 10^{\mathrm{a}}, 12^{\mathrm{a}}, 14^{\mathrm{a}}, 16^{\mathrm{a}}$, $18^{\mathrm{a}}$ e $20^{\mathrm{a}}$ semana. Dentro de cada semana foram selecionados, para cada madeira amazônica: 12 corpos de provas submetidos ao ataque do fungo de podridáo branca; 12 corpos de corpos de prova submetidos ao ataque do fungo de podridáo parda; 12 corpos de prova do bloco de correçáo. Os corpos de prova selecionados foram submetidos à limpeza, para remoção do micélio aderido, e dispostos em estufa de circulação forçada de ar, sob condiçóes citadas, até obtenção de massa constante.

Em cada semana de amostragem foram determinadas as perdas de massa, de cada espécie amazônica, conforme critérios estabelecidos pelo procedimento D2017 da ASTM (2005). Subsequentemente, foram obtidos os parâmetros colorimétricos por meio de um espectrofotocolorímetro com resolução de $3 \mathrm{~nm}$ e dotado de uma esfera integradora de refletância difusa. Utilizou-se iluminante D65, composto de uma lâmpada de xenônio, o qual simula a radiação solar diurna, com um ângulo de observação de $10^{\circ} \mathrm{em}$ temperatura ambiente.

Antecedendo as análises procedeu-se à calibração do colorímetro com auxílio de duas referências fornecidas pelo equipamento, uma com $L^{*}=100$ (branco total) e outra com $\mathrm{L}^{*}=0$ (preto total).

A metodologia adotada para a determinação dos parâmetros colorimétricos L* (luminosidade), a* (coordenada verde-vermelho) e b* (coordenada amarelo-azul) foi o sistema CIELab, descrito por Camargos e Gonçalez (2001), sendo os mesmos obtidos diretamente no mostrador digital do espectrofotocolorímetro. Adicionalmente, determinouse a variação total da cor (Equaçáo 1), de acordo com o procedimento D2244 da ASTM (2009). Realizaram-se 10 leituras dos parâmetros colorimétricos, na seção transversal $(2,5 \times 2,5 \mathrm{~cm})$, para cada corpo de prova, sendo utilizado o valor médio dessas mediçóes.

$$
\Delta E=\sqrt{\Delta L^{*}+\Delta a^{*}+\Delta b^{*}}
$$

em que: $\Delta \mathrm{E}=$ variação total da cor; $\Delta \mathrm{L}^{*}=$ variação total do parâmetro colorimétrico $\mathrm{L}^{*} ; \Delta \mathrm{a}^{*}=$ variação total do parâmetro colorimétrico $\mathrm{a}^{*} ; \Delta \mathrm{b}^{*}=$ variação total do parâmetro colorimétrico $\mathrm{b}^{*}$.

Os dados de perda de massa e dos parâmetros colorimétricos foram analisados segundo um delineamento inteiramente casualizado, com 12 repetiçóes para cada um dos 6 tratamentos, submetidos à análise de variância e as médias comparadas pelo teste de LSD de Fischer ao nível de 5\% de probabilidade.

Adicionalmente, definiram-se modelos estatísticos, pelo método stepwise, para prediçáo da perda de massa em funçáo dos parâmetros colorimétricos das madeiras deterioradas pelos fungos apodrecedores (Equação 2). Realizou-se a seleção dos modelos estatísticos com base nos seguintes critérios: maior coeficiente de determinação ajustado $\left(\mathrm{R}^{2} \mathrm{aj}.\right)$, menor erro padrão de estimativa (Syx) e maior valor de $\mathrm{F}$ calculado (Fcalc.). 


$$
\left.P M=f\left(P C ; P C^{2} ; \frac{1}{P C} ; \sqrt{P C} ; \operatorname{Ln}(P C)\right) \quad \text { (Equação } 2\right)
$$

em que: $\mathrm{PM}=$ perda de massa; $\mathrm{PC}=$ parâmetro colorimétrico.

Todos os procedimentos estatísticos foram realizados com auxílio do software Statistical Analysis System - SAS.

\section{RESULTADOS}

De modo geral, independente do período e da espécie amazônica, os parâmetros colorimétricos $\mathrm{L}^{*}, \mathrm{a}^{*}$ e b* apresentaram valores significativamente distintos em função do ataque dos fungos de podridáo branca e parda (Tabelas $1,2$ e 3$)$.
Para a madeira de marupá verificou-se que a exposição ao fungo de podridáo branca ocasionou um acréscimo significativo nas coordenadas $\mathrm{a}^{*} \mathrm{eb}^{*}$, enquanto o $\mathrm{L}^{*}$ apresentou-se, de certo modo, estável. Com relação ao ataque proporcionado pelo fungo de podridão parda, os três parâmetros colorimétricos básicos apresentaram alteração significativa na pigmentação, em que $\mathrm{L}^{*}$ e b* reduziram e a* aumentou.

Nas amostras de jequitibá submetidas ao fungo de podridão branca foi constatado que os parâmetros $L^{*}$ e b* apresentaram valores significativamente acrescidos. Por sua vez, apesar dos valores da coordenada a*, antes e após a ação do fungo, manterem-se, de certa maneira, inalterados, verificouse um decréscimo de cerca de $25 \%$ na quarta, sexta e décima

Tabela 1 - Perda de massa e alteração dos parâmetros colorimétricos $\left(L^{*}=\right.$ luminosidade; $a^{*}=$ coordenada vermelho-verde; $b^{*}=$ coordenada amareloazul) da madeira de Simarouba amára (marupá) submetida ao ataque dos fungos apodrecedores Trametes versicolor (podridão branca) e Gloeophyllum trabeum (podridão parda).

\begin{tabular}{|c|c|c|c|c|c|c|c|c|}
\hline \multirow{2}{*}{ Período } & \multicolumn{2}{|c|}{$L^{*}$} & \multicolumn{2}{|c|}{$a^{*}$} & \multicolumn{2}{|c|}{$b^{*}$} & \multicolumn{2}{|c|}{ Perda de massa } \\
\hline & Podridão branca & Podridão parda & Podridão branca & Podridão parda & Podridão branca & Podridão parda & Podridão branca & Podridão parda \\
\hline 0 & $69,0 \pm 1,2 d^{*}$ & $69,0 \pm 1,2 \mathrm{a}$ & $4,5 \pm 0,3 \mathrm{a}$ & $4,5 \pm 0,3 a$ & $22,8 \pm 1,3 \mathrm{a}$ & $22,8 \pm 1,3$ cde & $0 \pm 0 \mathrm{a}$ & $0 \pm 0 \mathrm{a}$ \\
\hline 1 & $66,2 \pm 1,5$ ab $A$ & $62,3 \pm 5,5$ b A & $6,8 \pm 0,5 \mathrm{~b} \mathrm{~A}$ & $4,97 \pm 0,5 a b B$ & $29,9 \pm 1,0$ bc $A$ & $23,5 \pm 1,1$ abcde $B$ & $1,6 \pm 0,4$ a $A$ & $3,1 \pm 0,5$ b B \\
\hline 2 & $65,2 \pm 0,7$ a $A$ & $50,2 \pm 4,1$ c B & $7,1 \pm 0,4 \mathrm{~b} \mathrm{~A}$ & $5,31 \pm 0,6$ b B & $29,9 \pm 1,0$ bc $A$ & $24,5 \pm 2,0 a b c B$ & $7,6 \pm 1,0 \mathrm{~b} \mathrm{~A}$ & $19,2 \pm 1,0$ c B \\
\hline 3 & $71,7 \pm 1,0$ e $A$ & $48,7 \pm 3,9$ c B & $7,2 \pm 0,4$ b A & $5,2 \pm 0,7$ b B & $29,6 \pm 1,0$ b A & $23,6 \pm 1,4$ abcde $B$ & $13,3 \pm 2,1$ c $A$ & $25,7 \pm 2,6 \mathrm{~dB}$ \\
\hline 4 & $70,6 \pm 1,5$ e $A$ & $47,1 \pm 5,8 \mathrm{~cd} \mathrm{~B}$ & $7,3 \pm 0,5 \mathrm{~b} \mathrm{~A}$ & $6,5 \pm 1,0 \mathrm{c} \mathrm{A}$ & $32,1 \pm 1,1 \mathrm{~d} A$ & $25,35 \pm 2,8$ a $B$ & $16,9 \pm 2,3 \mathrm{~d} A$ & $29,6 \pm 3,8$ e $B$ \\
\hline 6 & $68,6 \pm 1,0 \mathrm{~d} A$ & $48,0 \pm 6,8 \mathrm{~cd} \mathrm{~B}$ & $8,3 \pm 0,5 \mathrm{c} \mathrm{A}$ & $6,8 \pm 0,9 \mathrm{c} \mathrm{B}$ & $31,0 \pm 1,3 \mathrm{~cd} A$ & $24,9 \pm 3,4$ ab B & $24,8 \pm 3,8$ e $A$ & $35,4 \pm 2,3 \mathrm{fB}$ \\
\hline 8 & $69,1 \pm 3,4 \mathrm{~d} A$ & $44,1 \pm 5,6 \mathrm{~d} B$ & $8,7 \pm 0,6$ c A & $7,0 \pm 0,8 \mathrm{~cd} \mathrm{~B}$ & $30,9 \pm 1,2 \mathrm{bcd} A$ & $23,3 \pm 2,7$ bcde $B$ & $35,9 \pm 3,8 f A$ & $44,3 \pm 5,6 \mathrm{~g} \mathrm{~B}$ \\
\hline 10 & $66,4 \pm 1,0$ ab $A$ & $38,6 \pm 6,7$ e $B$ & $9,8 \pm 0,5$ de $A$ & $7,7 \pm 1,0$ de $B$ & $33,4 \pm 1,4$ e $A$ & $22,0 \pm 2,9 \operatorname{defg} B$ & $43,0 \pm 5,3 \mathrm{~g} \mathrm{~A}$ & $47,1 \pm 3,6$ h B \\
\hline 12 & $67,9 \pm 1,0 \mathrm{~cd} A$ & $37,7 \pm 2,7$ e $B$ & $9,6 \pm 0,5 \mathrm{~d} A$ & $7,9 \pm 1,0$ ef $B$ & $34,8 \pm 1,4$ f $A$ & $22,4 \pm 1,2$ def $B$ & $51,2 \pm 5,0$ h A & $54,3 \pm 3,1 \mathrm{i} A$ \\
\hline 14 & $66,8 \pm 1,4$ bc $A$ & $39,3 \pm 3,4$ e $B$ & $10,1 \pm 1,0$ def $A$ & $8,9 \pm 1,3 \mathrm{~g} \mathrm{~B}$ & $34,6 \pm 1,7 \mathrm{fA}$ & $23,7 \pm 2,3$ abcd B & $56,2 \pm 2,4$ i A & $54,6 \pm 4,0$ i A \\
\hline 16 & $69,0 \pm 1,3 \mathrm{~d} A$ & $35,7 \pm 4,0$ ef $B$ & $10,2 \pm 1,0$ ef $A$ & $8,8 \pm 0,6 \mathrm{~g} \mathrm{~B}$ & $36,4 \pm 2,2 \mathrm{~g} \mathrm{~A}$ & $20,8 \pm 2,3 \mathrm{fg} B$ & $56,6 \pm 6,3$ i A & $57,9 \pm 4,4$ j A \\
\hline 18 & $66,5 \pm 1,3$ ab $A$ & $37,6 \pm 4,7$ e $B$ & $10,1 \pm 1,0 \operatorname{def} A$ & $8,6 \pm 0,8 \mathrm{fg} \mathrm{B}$ & $35,9 \pm 1,4 \mathrm{~g} \mathrm{~A}$ & $21,7 \pm 2,5$ efg $B$ & $62,0 \pm 7,5 \mathrm{j} A$ & $60,3 \pm 4,6$ j $A$ \\
\hline 20 & $68,2 \pm 1,7 \mathrm{~d} A$ & $33,1 \pm 6,4 \mathrm{f} B$ & $10,4 \pm 0,8 f A$ & $8,3 \pm 1,1$ efg $B$ & $35,6 \pm 1,2 \mathrm{fg} A$ & $20,0 \pm 4,0 \mathrm{~g} \mathrm{~B}$ & $69,4 \pm 6,4 \mathrm{k} \mathrm{B}$ & $64,7 \pm 3,5 \mathrm{k} \mathrm{A}$ \\
\hline
\end{tabular}

*Médias seguidas na horizontal por uma mesma letra maiúscula ou na vertical por uma mesma letra minúscula não diferem estatisticamente a $5 \%$ de probabilidade pelo teste LSD de Fischer.

Tabela 2 - Perda de massa e alteração dos parâmetros colorimétricos $\left(L^{*}=\right.$ luminosidade; $a^{*}=$ coordenada vermelho-verde; $b^{\star}=$ coordenada amareloazul) da madeira de Cariniana micrantha (jequitibá) submetida ao ataque dos fungos apodrecedores Trametes versicolor (podridão branca) e Gloeophyllum trabeum (podridão parda).

\begin{tabular}{|c|c|c|c|c|c|c|c|c|}
\hline \multirow{2}{*}{ Período } & \multicolumn{2}{|c|}{$L^{*}$} & \multicolumn{2}{|c|}{$a^{*}$} & \multicolumn{2}{|c|}{$\mathrm{b}^{\star}$} & \multicolumn{2}{|c|}{ Perda de massa } \\
\hline & Podridão branca & Podridão parda & Podridão branca & Podridão parda & Podridão branca & Podridão parda & Podridão branca & Podridão parda \\
\hline 0 & $52,7 \pm 1,4 a^{*}$ & $52,7 \pm 1,4 \mathrm{a}$ & $7,9 \pm 0,4$ def & $7,9 \pm 0,4 \mathrm{c}$ & $15,1 \pm 0,8 \mathrm{a}$ & $15,1 \pm 0,8 \mathrm{~cd}$ & $0 \pm 0 \mathrm{a}$ & $0 \pm 0 \mathrm{a}$ \\
\hline 1 & $53,2 \pm 3,1$ a $A$ & $50,2 \pm 2,4$ a B & $7,7 \pm 0,4$ cde $B$ & $9,4 \pm 0,9$ a $A$ & $21,1 \pm 1,0 \mathrm{~b} \mathrm{~A}$ & $21,4 \pm 1,3$ a $A$ & $0 \pm 0$ a $A$ & $0,2 \pm 0,1 \mathrm{a} B$ \\
\hline 2 & $58,3 \pm 2,5 \mathrm{~b} \mathrm{~A}$ & $42,9 \pm 4,4$ b B & $7,5 \pm 1,0$ bcd B & $9,4 \pm 1,0$ a $A$ & $22,9 \pm 1,8 \mathrm{~cd} \mathrm{~A}$ & $21,0 \pm 2,0$ a B & $3,5 \pm 0,8$ a B & $2,0 \pm 1,1$ a $A$ \\
\hline 3 & $59,0 \pm 2,9$ b A & $42,9 \pm 6,2$ b B & $8,2 \pm 0,9$ ef $A$ & $8,9 \pm 0,8$ ab $A$ & $23,6 \pm 1,3$ de $A$ & $21,7 \pm 2,2$ a $A$ & $9,6 \pm 0,9$ b B & $6,5 \pm 1,6 \mathrm{~b} \mathrm{~A}$ \\
\hline 4 & $64,4 \pm 2,0 \mathrm{cA}$ & $43,9 \pm 4,5$ b B & $5,9 \pm 0,6$ a B & $9,6 \pm 1,2$ a $A$ & $21,8 \pm 1,1 \mathrm{bc} A$ & $22,7 \pm 1,3$ a $A$ & $13,5 \pm 1,7$ b B & $11,0 \pm 1,0 \mathrm{c} \mathrm{A}$ \\
\hline 6 & $67,2 \pm 0,9$ de $A$ & $37,7 \pm 2,7$ с B & $6,0 \pm 0,9$ a B & $9,3 \pm 0,6$ a $A$ & $24,5 \pm 2,4$ e $A$ & $18,5 \pm 1,0$ b B & $21,5 \pm 3,1$ c B & $17,5 \pm 1,3 \mathrm{~d} A$ \\
\hline 8 & $64,5 \pm 1,4$ c A & $33,5 \pm 2,9 \mathrm{~d} \mathrm{~B}$ & $7,1 \pm 0,5$ bc $B$ & $9,2 \pm 0,3$ a $A$ & $27,3 \pm 1,5 f \mathrm{~A}$ & $18,9 \pm 1,5$ b B & $28,4 \pm 3,2 \mathrm{~d} \mathrm{~B}$ & $21,9 \pm 2,9$ e $A$ \\
\hline 10 & $64,2 \pm 2,5 \mathrm{c} \mathrm{A}$ & $32,4 \pm 2,4$ de $B$ & $8,5 \pm 1,2 f A$ & $9,1 \pm 0,7$ a $A$ & $27,6 \pm 1,9+A$ & $17,8 \pm 1,4$ b B & $34,5 \pm 3,5$ е $B$ & $29,4 \pm 2,3 f A$ \\
\hline 12 & $69,6 \pm 1,5 \mathrm{f} A$ & $29,3 \pm 2,3$ ef $B$ & $6,0 \pm 0,5$ a B & $8,9 \pm 0,8$ ab $A$ & $24,6 \pm 1,3$ e $A$ & $16,0 \pm 1,5$ c B & $42,5 \pm 3,0 \mathrm{fB}$ & $34,3 \pm 3,4 \mathrm{~g} \mathrm{~A}$ \\
\hline 14 & $64,6 \pm 1,4 \mathrm{cA}$ & $32,1 \pm 3,2$ de $B$ & $7,0 \pm 0,3$ b B & $7,7 \pm 0,4 \mathrm{~cd} \mathrm{~A}$ & $28,6 \pm 2,0+A$ & $15,4 \pm 1,4 \mathrm{~cd} \mathrm{~B}$ & $49,2 \pm 4,0 \mathrm{~g} \mathrm{~B}$ & $36,0 \pm 4,5 \mathrm{~g} \mathrm{~A}$ \\
\hline 16 & $67,2 \pm 2,4 \mathrm{~d} A$ & $26,9 \pm 4,1 \mathrm{fg} B$ & $7,7 \pm 0,5$ cde $A$ & $8,2 \pm 0,6$ bc $A$ & $30,2 \pm 1,6 \mathrm{~g} \mathrm{~A}$ & $14,7 \pm 2,1$ cde $B$ & $47,4 \pm 4,8 \mathrm{~g} \mathrm{~A}$ & $43,9 \pm 4,9$ h A \\
\hline 18 & $67,3 \pm 1,3$ de $A$ & $27,4 \pm 3,6 \mathrm{fg} B$ & $8,2 \pm 0,6$ ef $A$ & $7,5 \pm 1,4 \mathrm{~cd} A$ & $31,2 \pm 1,9 \mathrm{~g} \mathrm{~A}$ & $13,9 \pm 2,9$ de $B$ & $48,7 \pm 12,7 \mathrm{~g} \mathrm{~A}$ & $47,6 \pm 6,4$ i A \\
\hline 20 & $69,1 \pm 1,2$ ef $A$ & $26,2 \pm 2,0 \mathrm{~g} \mathrm{~B}$ & $7,5 \pm 0,9$ bcd $A$ & $7,2 \pm 0,9 \mathrm{~d} \mathrm{~A}$ & $31,0 \pm 2,1 \mathrm{~g} \mathrm{~A}$ & $13,2 \pm 1,7$ e $B$ & $48,7 \pm 11,2 \mathrm{~g} \mathrm{~A}$ & $49,0 \pm 6,4$ i A \\
\hline
\end{tabular}

*Médias seguidas na horizontal por uma mesma letra maiúscula ou na vertical por uma mesma letra minúscula não diferem estatisticamente a $5 \%$ de probabilidade pelo teste LSD de Fischer. 
Tabela 3 - Perda de massa e alteração dos parâmetros colorimétricos $\left(L^{*}=\right.$ luminosidade; $a^{*}=$ coordenada vermelho-verde; $b^{*}=$ coordenada amareloazul) da madeira de Dipteryx odorata (cumaru) submetida ao ataque dos fungos apodrecedores Trametes versicolor (podridão branca) e Gloeophyllum trabeum (podridão parda).

\begin{tabular}{lllllllll}
\hline \multirow{2}{*}{ Período o } & \multicolumn{3}{c}{$\mathrm{L}^{*}$} & \multicolumn{2}{c}{$\mathrm{a}^{*}$} & \multicolumn{2}{c}{ Perda de massa } \\
\cline { 2 - 9 } & Podridão branca & Podridão parda & Podridão branca & Podridão parda & Podridão branca & Podridão parda & Podridão branca & Podridão parda \\
\hline 0 & $48,2 \pm 4,2$ a* & $48,2 \pm 4,2$ a & $9,1 \pm 1,0$ dfgh & $9,1 \pm 1,0$ a & $18,7 \pm 0,9$ ab & $18,7 \pm 0,9$ c & $0 \pm 0$ a & $0 \pm 0$ a \\
1 & $41,4 \pm 2,6$ cd A & $45,7 \pm 5,2$ ab A & $10,6 \pm 0,5$ a A & $6,6 \pm 0,5$ cdef B & $18,2 \pm 1,9$ a B & $21,3 \pm 2,2$ a A & $0 \pm 0$ a A & $0,4 \pm 0,2$ ab B \\
2 & $41,2 \pm 1,3$ d B & $44,1 \pm 3,2$ bc A & $9,6 \pm 0,7$ bcdef A & $6,7 \pm 0,5$ cdef B & $18,6 \pm 1,0$ ab B & $21,0 \pm 2,0$ ab A & $0 \pm 0$ a A & $0,4 \pm 0,2$ ab B \\
3 & $44,9 \pm 1,5$ b A & $42,1 \pm 2,6$ c B & $8,9 \pm 0,4$ efgh A & $6,8 \pm 0,6$ bcde B & $20,2 \pm 1,3$ cd A & $19,7 \pm 1,3$ bc A & $0 \pm 0$ a A & $0 \pm 0$ a A \\
4 & $43,4 \pm 2,2$ bc A & $42,2 \pm 1,4$ c A & $9,4 \pm 1,3$ bcdefg A & $6,2 \pm 0,6$ fg B & $20,5 \pm 1,7$ cde A & $18,5 \pm 0,9$ c B & $1,1 \pm 0,3$ d A & $1,2 \pm 0,3$ bc A \\
6 & $41,8 \pm 1,9$ cd A & $33,1 \pm 3,8$ d B & $10,0 \pm 0,7$ ab A & $7,2 \pm 0,3$ b B & $20,8 \pm 1,3$ cdef A & $15,0 \pm 2,0$ d B & $1,0 \pm 0,4$ cd A & $1,1 \pm 0,3$ bc A \\
8 & $41,0 \pm 1,3$ d A & $28,9 \pm 4,4$ e B & $9,7 \pm 0,6$ bcde A & $7,1 \pm 0,4$ bc B & $21,1 \pm 1,1$ cdef A & $13,4 \pm 2,9$ e B & $0,5 \pm 0,5$ b A & $1,7 \pm 0,8$ c B \\
10 & $40,4 \pm 1,3$ d A & $27,7 \pm 1,1$ ef B & $8,5 \pm 0,7$ h A & $7,0 \pm 0,3$ bcd B & $19,9 \pm 1,1$ bc A & $12,4 \pm 0,9$ e B & $0,8 \pm 0,4$ bc A & $1,6 \pm 0,5$ c B \\
12 & $43,7 \pm 1,2$ b A & $28,4 \pm 0,8$ e B & $9,1 \pm 0,8$ cdefgh A & $6,3 \pm 0,4$ efg B & $21,5 \pm 2,0$ def A & $11,9 \pm 0,4$ ef B & $1,2 \pm 0,4$ de A & $2,7 \pm 1,3$ d B \\
14 & $43,8 \pm 2,8$ b A & $25,2 \pm 2,2$ fg B & $9,7 \pm 0,9$ bcd A & $6,5 \pm 0,6$ def B & $21,8 \pm 1,2$ ef A & $11,0 \pm 0,8$ fg B & $1,4 \pm 0,5$ e A & $4,3 \pm 0,9$ ef B \\
16 & $45,2 \pm 0,9$ b A & $25,1 \pm 1,6$ fg B & $8,8 \pm 0,4$ fgh A & $6,5 \pm 0,6$ def B & $21,6 \pm 1,4$ def A & $10,8 \pm 1,3$ fg B & $0,6 \pm 0,3$ b A & $4,2 \pm 1,3$ ef B \\
18 & $43,3 \pm 2,6$ bc A & $25,0 \pm 1,6$ fg B & $8,9 \pm 0,7$ gh A & $6,3 \pm 0,4$ efg B & $21,7 \pm 1,9$ ef A & $10,4 \pm 0,9$ g B & $0,9 \pm 0,6$ bcd A & $3,8 \pm 1,7$ e B \\
20 & $43,6 \pm 1,4$ bc A & $24,6 \pm 2,8$ g B & $9,8 \pm 0,8$ abc A & $6,0 \pm 0,6$ g B & $22,1 \pm 1,2$ f A & $9,9 \pm 1,5 \mathrm{~g} \mathrm{~B}$ & $1,1 \pm 0,6$ de A & $5,1 \pm 2,3$ fg B \\
\hline
\end{tabular}

*Médias seguidas na horizontal por uma mesma letra maiúscula ou na vertical por uma mesma letra minúscula não diferem estatisticamente a $5 \%$ de probabilidade pelo teste LSD de Fischer.

segunda semana. Ao considerar o ataque do fungo de podridão parda constatou-se que os três parâmetros colorimétricos básicos da madeira de jequitibá foram significativamente reduzidos.

Em relação à madeira de cumaru foi possível verificar que tanto para a deterioração causada pelo fungo de podridão branca, quanto de podridáo parda, o número de parâmetros colorimétricos significativamente modificados foi reduzido, ao realizar a comparaçáo com as madeiras de marupá e jequitibá. Para a podridão branca, verificou-se que, apenas, a coordenada $\mathrm{b}^{*}$ foi influenciada, sendo esta significativamente elevada com a exposição, enquanto sob o ataque do fungo de podridão parda constatou-se que os parâmetros $\mathrm{L}^{*} \mathrm{e} \mathrm{b}^{*}$ apresentaram valores significativamente reduzidos.

As curvas de refletância das madeiras deterioradas de marupá (Figura 1) e jequitibá (Figura 2) apresentaram comportamento semelhante, em que a podridão branca e parda proporcionaram coloraçôes mais claras e escuras, ou seja, refletindo mais e menos a luz incidente, respectivamente, em comparação as mesmas madeiras não atacadas. Por sua vez, as curvas de refletância da madeira de cumaru (Figura 3) apresentaram um mesmo comportamento, independente do fungo apodrecedor, em que as coloraçóes dos corpos de prova deteriorados foram mais escuras, de maneira a refletir menos a luz incidente.

Nas Figuras 4 a 6 pode-se verificar que a magnitude do parâmetro colorimétrico $\Delta \mathrm{E}$ foi maior no ataque proporcionado pelo fungo de podridão parda, independente da espécie amazônica.

Mediante análise dos parâmetros estatísticos (Tabela 4), verificou-se que mais de um parâmetro colorimétrico apresentou correlaçáo com a perda de massa para cada fungo apodrecedor e espécie amazônica, exceto para a podridão branca na madeira de cumaru.

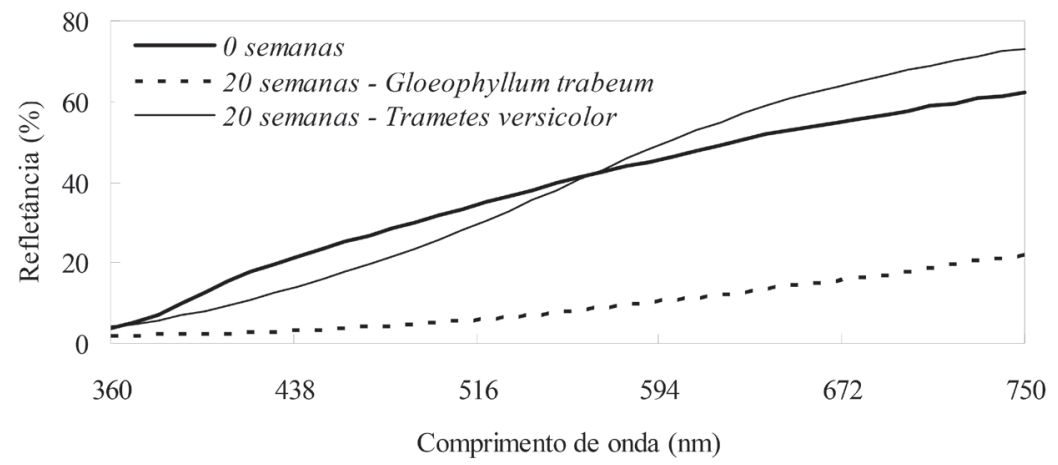

Figura 1 - Curvas de refletância da madeira de Simarouba amara (marupá) antes e após o ataque dos fungos apodrecedores Trametes versicolor (podridão branca) e Gloeophyllum trabeum (podridão parda). 


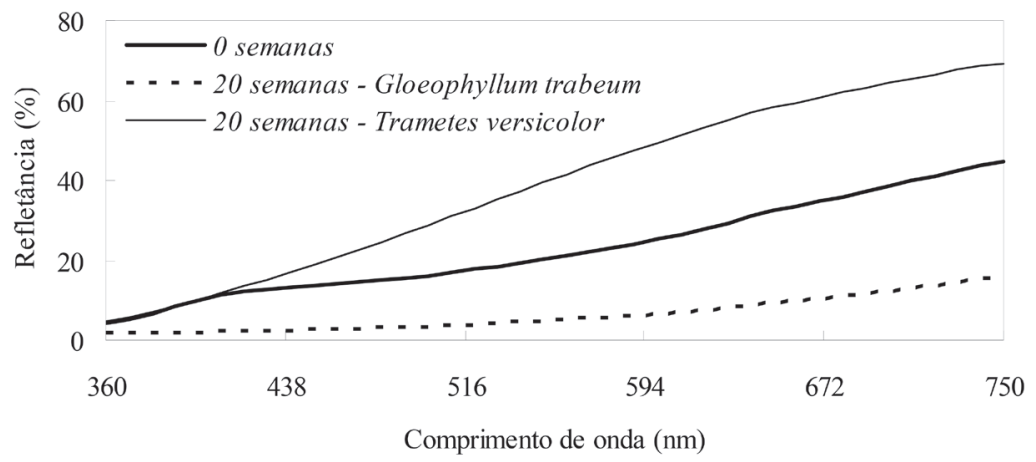

Figura 2 - Curvas de refletância da madeira de Cariniana micrantha (jequitibá) antes e após o ataque dos fungos apodrecedores Trametes versicolor (podridão branca) e Gloeophyllum trabeum (podridão parda).

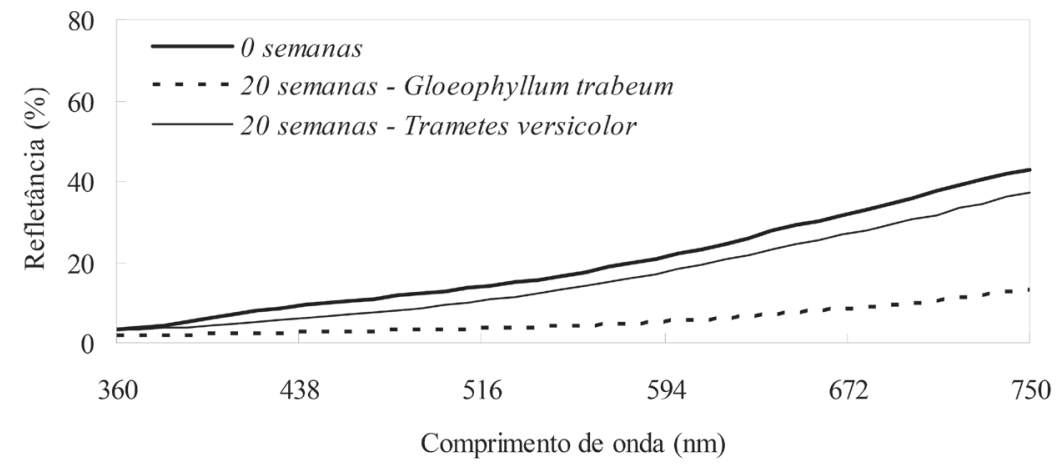

Figura 3 - Curvas de refletância da madeira de Dipteryx odorata (cumaru) antes e após o ataque dos fungos apodrecedores Trametes versicolor (podridão branca) e Gloeophyllum trabeum (podridão parda).

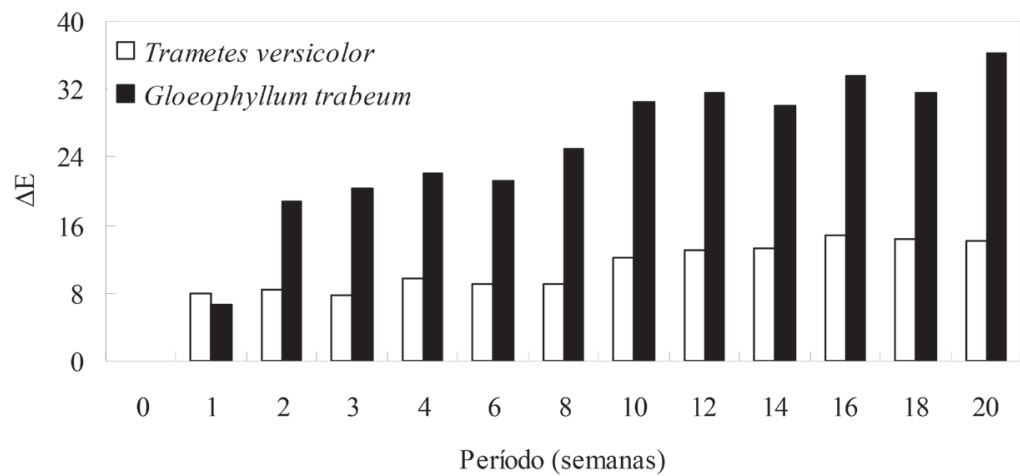

Figura 4 - Variação total da cor $(\Delta \mathrm{E})$ da madeira de Simarouba amara (marupá) submetida ao ataque dos fungos apodrecedores Trametes versicolor (podridão branca) e Gloeophyllum trabeum (podridão parda). 


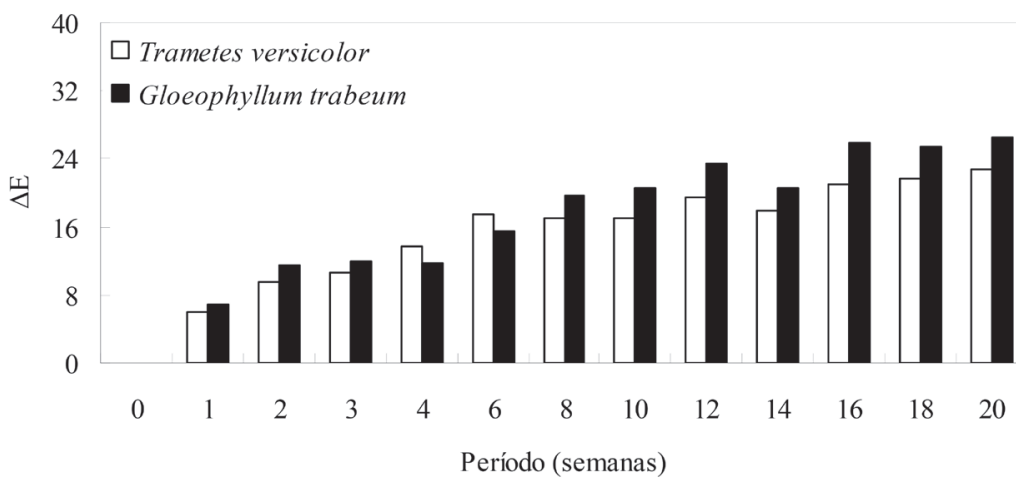

Figura 5 - Variação total da cor $(\Delta \mathrm{E})$ da madeira de Cariniana micrantha (jequitibá) submetida ao ataque dos fungos apodrecedores Trametes versicolor (podridão branca) e Gloeophyllum trabeum (podridão parda).

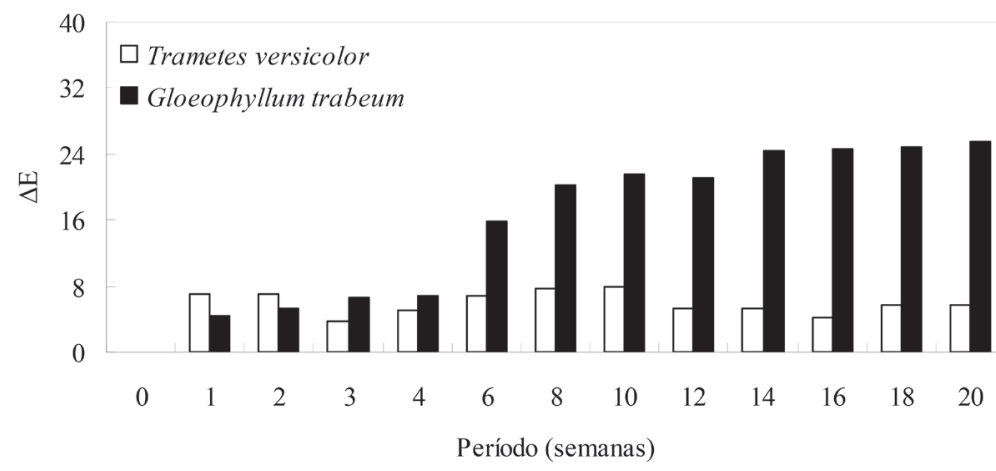

Figura 6 - Variação total da cor $(\Delta \mathrm{E})$ da madeira de Dipteryx odorata (cumaru) submetida ao ataque dos fungos apodrecedores Trametes versicolor (podridão branca) e Gloeophyllum trabeum (podridão parda).

Tabela 4 - Correlação entre a perda de massa (PM) e as alterações dos parâmetros colorimétricos ( $\mathrm{L}^{*}=$ luminosidade; $\mathrm{a}^{*}=$ coordenada vermelho-verde; $\mathrm{b}^{*}=$ coordenada amarelo-azul; $\Delta \mathrm{E}=$ variação total da cor) das madeiras de Simarouba amara (marupá), Cariniana micrantha (jequitibá) e Dipteryx odorata (cumaru) submetidas ao ataque dos fungos apodrecedores Trametes versicolor (podridão branca) e Gloeophyllum trabeum (podridão parda).

\begin{tabular}{|c|c|c|c|c|c|c|}
\hline & \multicolumn{2}{|c|}{ Marupá } & \multicolumn{2}{|c|}{ Jequitibá } & \multicolumn{2}{|c|}{ Cumaru } \\
\hline & Podridão branca & Podridão parda & Podridão branca & Podridão parda & Podridão branca & Podridão parda \\
\hline$P M \times L^{*}$ & $-0,15\left(0,63^{\mathrm{NS}}\right)$ & $-0,97\left(<0,01^{* *}\right)$ & $0,85\left(<0,01^{* *}\right)$ & $-0,95\left(<0,01^{* *}\right)$ & $-0,11\left(0,72^{\mathrm{NS}}\right)$ & $-0,87\left(<0,01^{* *}\right)$ \\
\hline PM $x a^{*}$ & $0,94\left(<0,01^{* \star}\right)$ & $0,96\left(<0,01^{\star *}\right)$ & $-0,05\left(0,86^{\mathrm{NS}}\right)$ & $-0,65\left(0,015^{\star}\right)$ & $-0,05\left(0,86^{\mathrm{NS}}\right)$ & $-0,54\left(0,05^{\mathrm{NS}}\right)$ \\
\hline$P M \times b^{\star}$ & $0,87\left(<0,01^{\star *}\right)$ & $-0,56\left(0,04^{\star}\right)$ & $0,88\left(<0,01^{\star *}\right)$ & $-0,77\left(<0,01^{\star *}\right)$ & $0,81\left(<0,01^{\star *}\right)$ & $-0,90\left(<0,01^{\star *}\right)$ \\
\hline $\mathrm{PM} \times \Delta \mathrm{E}$ & $0,87\left(<0,01^{\star \star}\right)$ & $0,97\left(<0,01^{\star \star}\right)$ & $0,91\left(<0,01^{\star \star}\right)$ & $0,94\left(<0,01^{\star \star}\right)$ & $0,24\left(0,43^{\mathrm{NS}}\right)$ & $0,89\left(<0,01^{\star \star}\right)$ \\
\hline
\end{tabular}

${ }^{* *}=$ significativo a $1 \%$ de probabilidade; * $=$ significativo a $5 \%$ de probabilidade; ${ }^{\text {NS }}=$ não significativo.

$\mathrm{L}^{*}$ e b* foram os principais parâmetros colorimétricos para predição da perda de massa das madeiras atacadas pelos fungos de podridáo parda e branca, de modo a proporcionar o escurecimento e amarelecimento das amostras, respectivamente (Tabela 5). Outro ponto a ser destacado foi a correlação significativa entre a $\Delta \mathrm{E}$ e a perda de massa ocasionada pelos fungos apodrecedores, exceto para a madeira de cumaru atacada pelo fungo de podridáo branca.

\section{DISCUSSÃO}

As alteraçôes significativas nos parâmetros colorimétricos $L^{*}, a^{*}$ e $b^{*}$ proporcionadas pelo ataque dos fungos apodrecedores podem ser justificadas pelos diferentes mecanismos enzimáticos que os fungos de podridão branca e parda apresentam. Em complemento, Okino et al. (2009) mencionaram que a análise colorimétrica de uma madeira submetida à exposição de fungos apodrecedores é influenciado 
pela presença de estruturas de lignina residual e, ou, modificação das hemiceluloses.

Com relação à madeira de marupá, apesar do acréscimo na coordenada $\mathrm{a}^{*}$, que atribui um avermelhamento, devese ressaltar que a coordenada $b^{*}$ foi o principal parâmetro responsável pela alteração colorimétrica da madeira deteriorada pelo fungo de podridão branca, e que seu acréscimo ocasionou o amarelecimento. Para o ataque do fungo de podridão parda, a interação entre os três parâmetros colorimétricos foi responsável pelo escurecimento da madeira de marupá, uma vez que os parâmetros $L^{*}$ e $b^{*}$ diminuíram, de modo a ocasionar o escurecimento e o menor amarelecimento, respectivamente $\mathrm{e}$, a coordenada $\mathrm{a}^{*}$ aumentou a pigmentação avermelhada da madeira deteriorada. Costa et al. (2011) ao submeterem a madeira de marupá aos mesmos fungos apodrecedores verificaram comportamento colorimétrico semelhante.

Quando considerada a madeira de jequitibá, verificou-se que o fungo de podridáo branca proporcionou um maior clareamento e amarelecimento, em razão do aumento dos parâmetros L* e b*, respectivamente. Okino et al. (2009) verificaram comportamento semelhante ao analisarem o efeito de Phanerochaete chrysosporium (podridáo branca) em seis clones de Hevea brasiliensis, em que a coordenada b*, num primeiro plano, e $\mathrm{L}^{*}$, num segundo plano, foram os mais modificados, enquanto a coordenada $\mathrm{a}^{*}$ permaneceu estável. Em relação à podridão parda, o parâmetro colorimétrico $\mathrm{L}^{*}$ foi o principal responsável pelo escurecimento da madeira deteriorada, visto que o decréscimo na magnitude das coordenadas $\mathrm{a}^{*}$ e $\mathrm{b}^{*}$, apenas, foi registrado a partir da segunda semana, sendo que na primeira semana ocorreu um aumento. Provavelmente, esse acréscimo inicial foi causado pelo contato dos micélios do fungo com a superfície da madeira, e posteriormente, com o progresso da difusão das hifas no interior da madeira foi estabelecido o decréscimo dos parâmetros.

Para a madeira de cumaru, a maior dificuldade de difusāo das hifas proporcionou alteraçôes colorimétricas menos acentuadas, sendo verificado um maior amarelecimento $\left(+\mathrm{b}^{*}\right)$ e, um maior escurecimento $\left(-L^{*}\right)$ e menor amarelecimento $\left(-b^{*}\right)$ nas amostras submetidas à podridão branca e parda, respectivamente. Tal resultado pode ser justificado em razão da elevada resistência natural da madeira de cumaru, a qual está relacionada à alta massa específica básica $\left(0,93 \mathrm{~g} \cdot \mathrm{cm}^{-3}\right)$, bem como ao elevado teor de extrativos (9,2\%) (Stangerlin 2012).

O escurecimento das amostras de marupá, jequitibá e cumaru atacadas pelo fungo de podridáo parda é explicado com base no mecanismo de deterioração, em que o mesmo ataca, preferencialmente, os polissacarídeos mantendo a lignina, teoricamente, intacta. Com relação às coloraçóes mais claras nas madeiras de marupá e jequitibá, proporcionadas pelo fungo de podridáo branca, tal resultado pode estar relacionado a uma característica de seletividade de deterioração da lignina. Apesar de alguns estudos apontarem o fungo Trametes versicolor como de ataque não-seletivo (Davis et al. 1994; Mohebby 2005), ou seja, de modo a proporcionar a despolimerização indistinta dos constituintes químicos, o mesmo, por vezes, pode apresentar predisposiçáo de ataque das fraçóes de lignina (Pandey e Navgeni 2007). Diferentemente, o escurecimento das amostras de cumaru deterioradas pelo fungo de podridáo branca pode ser justificável em razão do ataque simultâneo dos constituintes químicos, em especial da fração de holocelulose.

A maior $\Delta \mathrm{E}$ nas madeiras submetidas à podridáo parda é justificada pela acentuada variaçáo do parâmetro $L^{*}$. Adicionalmente, ressalta-se que ao considerar a coloraçáo inicial, antes do ataque fúngico, as madeiras mais claras (+ $\mathrm{L}^{*}$ ), no caso do marupá e jequitibá, apresentaram maior $\Delta \mathrm{E}$

Tabela 5 - Modelos de predição da perda de massa (PM) em função das alterações dos parâmetros colorimétricos $\left(L^{*}=\right.$ luminosidade; $b^{*}=$ coordenada amarelo-azul; $\Delta \mathrm{E}=$ variação total da cor) das madeiras de Simarouba amara (marupá), Cariniana micrantha (jequitibá) e Dipteryx odorata (cumaru) submetidas ao ataque dos fungos apodrecedores Trametes versicolor (podridão branca) e Gloeophyllum trabeum (podridão parda).

\begin{tabular}{|c|c|c|c|c|}
\hline Madeira x Fungo & Equação de regressão & $R^{2}$ aj. & Syx & Fcalc. \\
\hline \multirow{2}{*}{ Marupá x Podridão branca } & $P M=255,667-21,570^{\star}\left(b^{\star}\right)+0,451^{\star}\left(b^{\star 2}\right)$ & 0,85 & 9,48 & 34,60 ** \\
\hline & $P M=-1,219-1,494^{\star}(\Delta E)+0,416^{\star}\left(\Delta E^{2}\right)$ & 0,87 & 8,80 & $41,04^{\star \star}$ \\
\hline \multirow{2}{*}{ Marupá x Podridão parda } & $\sqrt{ } \mathrm{PM}=16,102-0,228^{*}\left(\mathrm{~L}^{*}\right)$ & 0,97 & 0,42 & $390,43^{* *}$ \\
\hline & $\sqrt{ } \mathrm{PM}=0,358+0,226 *(\Delta \mathrm{E})$ & 0,97 & 0,42 & $394,85^{* *}$ \\
\hline \multirow{2}{*}{ Jequitibá x Podridão branca } & $P M=-69,079+3,780^{*}\left(b^{\star}\right)$ & 0,76 & 9,74 & $38,31^{* \star}$ \\
\hline & $\mathrm{PM}=-2,111-0,122^{\star}(\Delta \mathrm{E})+0,116^{\star}\left(\Delta \mathrm{E}^{2}\right)$ & 0,88 & 6,81 & $45,47^{\star \star}$ \\
\hline \multirow{2}{*}{ Jequitibá x Podridão parda } & $P M=186,015-6,939 *\left(L^{\star}\right)+0,065^{\star}\left(L^{\star 2}\right)$ & 0,96 & 3,76 & $136,29 * *$ \\
\hline & $P M=-1,226-0,141^{*}(\Delta E)+0,077^{*}(\Delta E)$ & 0,95 & 3,87 & $128,08 * *$ \\
\hline Cumaru x Podridão branca & $P M=-18,602+6,381 * \operatorname{Ln}\left(b^{\star}\right)$ & 0,62 & 0,32 & $20,39 * *$ \\
\hline \multirow{2}{*}{ Cumaru x Podridão parda } & $P M=24,540-1,171^{\star}\left(L^{*}\right)+0,014^{*}\left(L^{* 2}\right)$ & 0,85 & 0,69 & $35,53^{\star *}$ \\
\hline & $P M=0,663-0,155^{\star}(\Delta E)+0,012^{\star}\left(\Delta E^{2}\right)$ & 0,87 & 0,65 & $40,45^{\star \star}$ \\
\hline
\end{tabular}

$\mathrm{R}^{2} \mathrm{aj} .=$ coeficiente de determinação ajustado; Syx = erro padrão da estimativa; Fcalc. = valor de $\mathrm{F}$ calculado; ** = significativo a $1 \%$ de probabilidade. 
quando comparada ao cumaru, madeira mais escura $\left(-\mathrm{L}^{*}\right)$, invariável ao fungo apodrecedor. Esse resultado corrobora com os relatados por Costa et al. (2011) e Baar e Gryc (2012) ao avaliarem a resistência natural à deterioração biológica $e$ à fotodescoloração, respectivamente, de madeiras tropicais. Diante das consideraçôes a cerca da $\Delta \mathrm{E}$ pode-se afirmar que quanto menor a sua magnitude, maior é a atribuição de resistência natural da madeira.

Ao avaliar a resistência natural da madeira de Tectona grandis a ação de dois fungos de podridão parda, Gloeophyllum trabeum e Antrodia sp., Kokutse et al. (2006) verificaram que o parâmetro $L^{*}$ apresentou correlação significativa a $1 \%$ com a perda de massa. Por sua vez, ao considerar as coordenadas a* e $\mathrm{b}^{*}$, os mesmos autores não obtiveram correlaçóes significativas, náo sendo possível o emprego de tais parâmetros como estimadores da durabilidade natural da madeira. Entretanto, no presente estudo, além do $L^{*}$, pode-se observar que as coordenadas $\mathrm{a}^{*} \mathrm{e} \mathrm{b}^{*}$ também colaboraram como indicadores do processo de biodeterioração das madeiras de marupá e jequitibá, por sua vez para o cumaru, apenas, a coordenada $b^{*}$.

Em relação aos fungos de podridão branca Trametes versicolor e Pycnoporus sanguineus, Kokutse et al. (2006) constataram que os parâmetros colorimétricos $L^{*}, a^{*}$ e b* da madeira de Tectona grandis foram significativamente correlacionadas com a perda de massa. Porém, verificouse no presente estudo que nem todos os parâmetros colorimétricos básicos apresentaram correlação com a perda de massa ao fungo de podridáo branca, em que adicional ao parâmetro $b^{*}$, apenas, o $a^{*}$ e o $L^{*}$ foram significativamente influenciados, considerando as madeiras de marupá e jequitibá, respectivamente.

A baixa correlação verificada entre a alteração do parâmetro colorimétrico $\mathrm{a}^{*}$ e a perda de massa das madeiras de cumaru, para ambos os fungos apodrecedores, e jequitibá, para o fungo de podridáo branca, pode estar relacionada à presença de compostos fenólicos. De acordo com Klumpers et al. (1994), Dellus et al. (1997 a, b) e Burtin et al. (1998) o parâmetro colorimétrico $\mathrm{a}^{*}$ apresenta estreita relaçáo com os compostos fenólicos de diferentes madeiras, sendo essa classe de extrativos, de maneira geral, a responsável pela durabilidade natural da madeira.

Adicionalmente, constata-se que as madeiras de cumaru e jequitibá foram as que apresentam os maiores valores iniciais para a coordenada a*, quando comparada ao marupá, podendo assim afirmar que a maior intensidade do respectivo parâmetro foi responsável pela maior resistência biológica das mesmas, corroborando com Gierlinger et al. (2003) e Amusant et al. (2004, 2008).

\section{CONCLUSÕES}

A caracterização da colorimetria da madeira mostrou-se eficaz para o monitoramento da deterioraçáo por fungos apodrecedores, bem como para diferenciação da podridão branca e parda, nos diferentes estágios.

As melhores prediçôes da resistência natural aos fungos de podridão branca e parda foram obtidas com os parâmetros $b^{*}$ e L*, respectivamente.

A $\Delta \mathrm{E}$ apresentou viabilidade de ser empregada como parâmetro auxiliar na estimativa da biodeterioração da madeira, em razáo de suas correlaçôes significativas com a perda de massa.

\section{AGRADECIMENTOS}

Os autores agradecem ao Conselho Nacional de Desenvolvimento Científico e Tecnológico (CNPq), pelo financiamento da pesquisa.

\section{BIBLIOGRAFIA CITADA}

Almeida, N.A.; Mendes, L.M.; Okino, E.Y.A.; Garlet, A.; Mori, F.A.; Mendes, R.F. 2012. Biodeterioraçáo de produtos à base da madeira de cedro australiano (Toona ciliata M. Roem. var. australis). Cerne, 18: 17-26.

Amusant, N.; Beauchene, J.; Fournier, M.; Janin, G.; Thevenon, M.F. 2004. Decay resistance in Dicorynia guianensis Amsh.: analysis of inter-tree and intra-tree variability and relations with wood colour. Annals of Forest Science, 61: 373-380.

Amusant, N.; Fournier, M.; Beauchene, J. 2008. Colour and decay resistance and its relationships in Eperua grandiflora. Annals of Forest Science, 65: 1-6.

American Society for Testing and Materials. 2005. ASTM D2017. Standard method for accelerated laboratory test of natural decay resistance for woods. ASTM International, West Conshohocken, PA, USA. 5 p.

American Society for Testing and Materials. 2009. ASTM D2244. Standard practice for calculation of color tolerances and color differences from instrumentally measured color coordinates. ASTM International, West Conshohocken, PA, USA. 11 p.

Baar, J.; Gryc, V. 2012. The analysis of tropical wood discoloration caused by simulated sunlight. European Journal of Wood and Wood Products, 70: 263-269.

Burtin, P.; Jay-Allemand, C.; Charpentier, J.P.; Janin, G. 1998. Natural wood colouring process in Juglans sp. (J. nigra, J. regia and hybrid J. nigra $23 \times J$. regia) depends on native phenolic compounds accumulated in the transition zone between sapwood and heartwood. Trees, 12: 258-264.

Camargos, J.A.A.; Gonçalez, J.C. 2001. A colorimetria aplicada como instrumento na elaboração de uma tabela de cores de madeira. Brasil Florestal, 71: 30-41. 
Carneiro, J.S.; Emmert, L.; Sternadt, G.H.; Mendes, J.C.; Almeida, G.F. 2009. Decay susceptibility of Amazon wood species from Brazil against white rot and brown rot decay fungi. Holzforschung, 63: 767-772.

Costa, M.A.; Costa, A.F. da; Pastore, T.C.M.; Braga, J.W.B.; Gonçalez, J.C. 2011. Caracterizaçấo do ataque de fungos apodrecedores de madeiras através da colorimetria e da espectroscopia de infravermelho. Ciência Florestal, 21: 567-577.

Davis, M.F.; Schroeder, H.A.; Maciel, G.E. 1994. Solid state ${ }^{13} \mathrm{C}$ nuclear magnetic resonance studies of wood decay. II White rot decay of paper birch. Holzforschung, 48: 186-192.

Dellus, V.; Scalbert, A.; Janin, G. 1997a. Polyphenols and colour of Douglas-fir heartwood. Holzforschung, 51: 291-295.

Dellus, V.; Scalbert, A.; Menard, C.; Michot, V. Penhoat, L.M. 1997b. Douglas-fir polyphenols and heartwoood formation. Phytochemistry, 45: 1573-1578.

Eaton, R.A.; Hale, M.D.C. 1993. Wood: decay, pests and protection. Chapman \& Hall, London, UK. 546 p.

Fengel, D.; Wegener, G. 1984. Wood: chemistry, ultrastructure, reactions. Walter de Gruyter, New York, NY, USA. 613 p.

Gierlinger, N.; Jacques, D.; Grabner, M.; Wimmer, R.; Schwanninger, M.; Rozenberg, P.; Pâques, L.E. 2003. Colour of larch heart wood and relationships to extractives and brown-rot decay resistance. Trees, 18: 102-108.

Gonçalez, J.C. 1993. Caracterization technologique de quatre espèces peu connues de la Forêt Amazonienne: anatomie, chimie, couleur, proprietés physiques et mécaniques. Tese de Doutorado, École Nationale du Gêne Rural des Eaux et des Forêts, Nancy. 444 p.

Klumpers J.; Scalbert, A.; Janin, G. 1994. Ellagitannins in European oak wood: polymerization during wood ageing. Phytochemistry, 36: 1249-1252.

Kokutse, A.D.; Stokes, A.; Baillères, H.; Kokou, K.; Baudasse, C. 2006. Decay resistance of Togolese teak (Tectona grandis L.f) heartwood and relationship with colour. Trees, 20: 219-223.

Moya, R.; Marín, J.D. 2011. Grouping of Tectona grandis (L.f.) clones using wood color and stiffness. New Forests, 42: 329-345.

Nishino Y.; Janin G.; Yamuda Y.; Kitano D. 2000. Relations between colorimetric values and densities of sapwood. Journal of Wood Science, 46: 267-272.
Martins, S.A.; Santos, C.M.T.; Gonçalez, J.C.; Camargos, J.A.A. 2011. Envelhecimento artificial acelerado por radiação ultravioleta de madeiras de Eucalyptus benthamii e Pinus caribaea var. hondurensis. Floresta, 41: 87-96.

Mohebby, B. 2005. Attenuated total reflection infrared spectroscopy of white-rot decayed beech wood. International Biodeterioration and Biodegradation, 55: 247-251.

Morais, F.M. de; Costa, A.F. da. 2007. Alteração da cor aparente de madeiras submetidas ao ataque de fungos apodrecedores. Revista Brasileira de Ciências Agrárias, 2: 44-50.

Okino, E.Y.A.; Pastore, T.C.M.; Camargos, J.A.A.; Alves, M.V.S.; Santos, P.H.O.; Teixeira, D.E.; Santana, M.A.E. 2009. Color variation of rubberwood clones and cypress infected by Gloeophyllum striatum and Phanerochaete chrysosporium. International Biodeterioration and Biodegradation, 63: 41-45.

Pandey, K.K.; Nagveni, H.C. 2007. Rapid characterization of brown and white rot degraded chir pine and rubberwood by FTIR spectroscopy. European Journal of Wood and Wood Products, 65: 477-481.

Santini, E.J. 1988. Biodeterioraçâo e preservação da madeira. CEPEF, Santa Maria, RS, Brasil. 125 p.

Souza, F.; Teles, R.F.; Ribeiro, P.G.; Camargos, A.M.; Del Menezzi, C.H.S. 2010. Resistência natural e alteração da cor da madeira de Machaerium scleroxylon Tul. submetida ao ataque de fungos apodrecedores. Scientia Forestalis, 38: 449-457.

Stangerlin, D.M. 2012. Monitoramento de propriedades de madeiras da Amazônia submetidas ao ataque de fungos apodrecedores. Tese de Doutorado, Universidade de Brasília, Brasília. 259 p.

Tovar, D.A.; Moya, R.; Tenorio, C. 2009. Wood color variation in undried and kiln-dried plantation-grown lumber of Vochysia guatemalensis. Maderas: Ciencia y tecnología, 11: 207-216.

Unsal, O.; Korkut, S.; Atik, C. 2003. The effect of heat treatment on some properties and colour in eucalyptus (Eucalyptus camaldulensis Dehn.) wood. Maderas: Ciencia y tecnología, 2: 145-152.

Valverde, J.C.; Moya, R. 2010. Efectos de la intemperie en el color de dos acabados aplicados en madera de Cedrela odorata y Carapa guianensis. Maderas: Ciencia y Tecnología, 12: 171-180.

Zabel, R.A.; Morrell, J.J. 1992. Wood microbiology: decay and its prevention. Academic Press, San Diego, CA, USA. 476 p.

Recebido em: 27/05/12

Aceito em: 14/09/2012 